\title{
Ostelamivir (Tamiflu) induced mania - a case report
}

\author{
SR Perera, R Wellaketiya
}

\section{Abstract}

Neuropsychiatric consequences of ostelamivir use have been reported worldwide. These include depression, mania, and exacerbation of symptoms in patients with schizophrenia. We report a case of ostelamivir induced mania in a 23-year-old young male patient, who recovered subsequently with treatment with antipsychotics. Given that the prescription of ostelamivir is now becoming more widespread, we recommend that clinicians be mindful about this possible side effect.

SLJ Psychiatry 2018; 9(1): 29-31

\section{Introduction}

Ostelamivir (Tamiflu) is an antiviral drug introduced following the pandemic of influenza A(H1N1) virus (1). It is well tolerated and has been shown to be effective in reducing the symptoms of influenza and is thus used globally (2-4).

Neuropsychiatric consequences of ostelamivir use have been reported worldwide. These include depression, mania, and exacerbation of symptoms in patients with schizophrenia $(5,6,7)$. There have been reports of many cases of abnormal behaviors and 70 deaths involving children and adolescents occurring in association with ostelamivir in Japan (8). We report a case of ostelamivir induced mania in a young male patient.

\section{Case report}

A 23-year old previously healthy young man was referred for a psychiatric assessment. He had shown a change in his behavior over the previous 48 hours. He was sleeping very little, and was pacing inside the rooms of his home, quoting aloud the words of prophets and teachers of religion at night. At other times he was heard singing aloud and dancing to the music on his radio, apparently oblivious of the fact as to how disturbing his behaviour was to those around him. He ate very little but was seemingly overenergetic and became quite irritable when others questioned him on his new ventures, which included writing several new books.

There was no past history of any behavior to suggest a mood disorder, and no family history of any psychiatric illness or self-harm. He had no noteworthy medical or surgical history, was a teetotaler and denied any use of any illicit substances either in the past or in the days leading up to this change in behaviour.
It was however noteworthy that his change of behavior started 24 hours after he took the initial dose of medication prescribed for his flu symptoms. This young man had been treated as an outpatient a few days previously, for fever, runny nose and body aches, which were very suggestive of an impending H1N1 influenza. The lack of facilities at the hospital meant that serology for influenza was not possible. With the benefit of recovery in mind, the treating physician had recommended that he be started on ostelamivir capsules, 75mg twice a day. Subse-quently his flu symptoms had begun to resolve soon after the second or third dose.

On mental state examination, he was disinhibited, overfamiliar and spoke at length, talking about his abilities and plans to write several new books. His mood was clearly elevated and he was grandiose in his thoughts. There were no delusions nor any thoughts or plans of violence or aggression to others, and he denied any perceptual disturbances. He was oriented in time, place and person but lacked insight into his condition.

There were no clinically significant findings on physical examination, and no positive ophthalmological findings or focal neurological signs. His blood tests were within normal range, and the urine toxicology screen was negative.

A diagnosis of a manic episode without psychotic symptoms, induced by ostelamivir was made. He was commenced on oral olanzapine $10 \mathrm{mg}$ and clonazepam 1 mg to be taken at night. He responded well to the above treatment and his sleep and level of activity improved markedly over the next 2 weeks, although his mood remained elevated for the next week. While continuing the recommended treatment regime, he gradually returned to his premorbid euthymic mood and premorbid level of functioning, during the next 6-7 weeks. 


\section{Discussion}

There are many debates with regards to the neuropsychiatric events in H1N1, and whether they are related to treatment with ostelamivir (9). There is some evidence to suggest that the neuropsychiatric events following H1N1 are no higher than in the general population, or those receiving other antiviral treatments (10). Others claim that neuropsychiatric consequences are a result of H1N1 itself and not because of ostelamivir use, while there are several case reports which strongly suggest a temporal association between ostelamivir use and the occurrence of neuropsychiatric events, in otherwise healthy patients (5-7).

Such neuropsychiatric events are reported to be commoner in adolescents and often occurred within 48 hours of commencing treatment; the three largest reported symptom categories have been abnormal behaviour, miscellaneous psychiatric events and delusions or perceptual disturbances $(9,10)$.

The influenza virus too has been implicated in neuropsychiatric disorders, with rates higher than in the general population $(9,11)$. Acute manifestations are associated with serious sequelae or death. Other manifestations include seizures, Reye's syndrome, acute necrotizing encephalopathy, transverse myelitis and aseptic meningitis and autoimmune conditions like Guillian-Barre Syndrome. There are reports of acute psychosis following influenza, and a case report of an ischemic stroke in a four year old male, associated with acute influenza (12). The more chronic viral induced neuropsychiatric events include Parkinsons disease and dementia (13). Two cases of mania induced by influenza B was reported in 1985 (14).

Ostelamivir related neuropsychiatric events of unusual behaviors, delusions and disturbances in perception have been reported, as well as case reports stating ostelamivir may induce mania and depression (5-8). Ostelamivir induced mania has been reported in Hong Kong (5).

The neuropsychiatric manifestations of ostelamivir are most likely due to its ability to alter dopamine metabolism in the medial prefrontal cortex. This has been reported following systemic administration in rats, and maybe associated with behavioural changes (15).

In our patient, a comprehensive history and investigation findings suggested that the mania was induced by ostelamivir, rather than due to any other organic cause. The absence of a past history of psychiatric illness, and the lack of a family history of mood disorders also supported this diagnosis.

What made this case most suggestive of being medication induced was the temporal relationship between the commencement of ostelamivir and the onset of symptoms, wherein the symptoms started approximately 24-36 hours after starting medication. In previously reported case reports too, the majority of neuropsychiatric manifestations appeared within the 48 hours of starting treatment. The quick resolution of symptoms also supports this diagnosis.

\section{Conclusions}

This is the first reported case of an ostelamivir related neuropsychiatric event in Sri Lanka. Since the prescription of ostelamivir has become more widespread, we recommend that clinicians be mindful about this possible side effect and monitor for any changes in behavior or mental state following prescription.

\section{Acknowledgements}

The authors extend their thanks to the patient and his family.

\section{Conflicts of interest}

None

\section{SR Perera, R Wellaketiya, Base Hospital Nikaweratiya Corresponding author: SR Perera}

Email: sayupsych@yahoo.com

http://orcid.org/0000-0001-6824-8991

\section{References}

1. Jamieson B, Jain R, Carleton B, Goldman RD. Use of Ostelamivir in childlren. Can Fam Physician 2009; 55: 1199-201.

2. Lindemann I, Jacobson $H$, Schuhbauer $\mathrm{D}$, et al. In vitro pharmacological selectivity profile of ostelamivir prodrug (Tamiflu) and active metabolite. Eur J Pharmacol 2010; 628: 6-10.

3. Bardsley-Elliot A, Noble S. Ostelamivir. Drugs 1999; 58: 851-60.

4. Nicholson KG, AnokiFY, Osterhaus AD, et al. Efficacy and safety of ostelamivir in treatment of acute influenza: a randomized controlled trial. Neuraminidase Inhibitor Flu Treatment Investigator Group. Lancet 2000: 355: 1845-50.

5. Chung S, Joung YS. Ostelamivir (Tamiflu) induced depressive episode in a female adolescent. Psychiatric Investig 2010; 7: 302-4.

6. Ho LNL, Chung JPY, Choy KLW. Ostelamivir-induced mania in apatient with H1N1. Am J Psychiatry 2010; 167; 350-50. 
7. Lan C, Liu C, Chen Y. Acute exacerbation of psychiatric symptoms during influenza treatment with ostelamivir in chronic schizophrenia. J Chin Med Assoc 2015; 78: 374-6.

8. Fuyuno I. Tamiflu side effects come under scrutiny. Nature 2007; 446: 358-9.

9. Toovey S, Rayner C, Prinssen E, et al. Assessment of neuropsychiatric adverse events in infleunza patients treated with ostelamivir: a comprehensive review. Drug Saf 2008; 31: 1097-114.

10 Smith JR , Sacks S. Incidence of neuropsychiatric adverse events in influenza patients treated with oseltamivir or no antiviral treatment. Int J Clin Pract 2009; 63: 596-605.

11. Manjunatha N, Math SB, Kulkarni GB, Chaturvedi SK. The neuropsychiatric aspects of influenza/swine flu: A selective review. Ind Psychiatry L 2011; 20: 83-90.
12. Masson A. Post-influenza acute psychosis in an old man. Ann Med Psychol (Paris) 1957; 115: 490-4.

13. Mattson MP. Infectious agents and age-related neurodegenerative disorders. Ageing Res Rev 2004; 3: 105-20.

14. Steinberg D, Hirsch SR, Marston SD, Reynolds K, Sutton RN. Influenza infection causing manic psychosis. Br J Psychiatry 1972; 120: 531-5.

15. So A, Ito M, Kusuhara H, Yamastugu K, Kanai M, Shibasaki M, et al. Limited brain distribution of (3R,4R,5S)-4-acetamido-5-amino-3-(1-ethylpropoxy)-1cyclohexene-1-carboxylate phosphate (Ro64-0802), a pharmacologically active form of ostelamivir, by effective efflux across the blood-brain barrier mediated by organic anion transporter 3b (Oat3/Slc22a8) and multi drug resistance-associated protein 4 (Mrp4/Abcc4). Drug Metab Dispos 2009; 37: 315-21. 